THU0577

DO RAYNAUD PHENOMENON NEGATIVE JUVENILE SYSTEMIC SCLERODERMA PATIENTS HAVE A DIFFERENT PATTERN OF ORGAN INVOLVEMENT AS RAYNAUD PHENOMENON POSITIVE PATIENTS?

I. Foeldvari ${ }^{1}$, J. Klotsche ${ }^{2}$, O. Kasapcopur ${ }^{3}$, A. Adrovic ${ }^{3}$, K. Torok ${ }^{3}$, V. Stanevicha ${ }^{3}$, M.T. Terreri ${ }^{3}$, E. Alexeeva ${ }^{3}$, M. Katsikas ${ }^{3}$, V. Smith ${ }^{3}$, F. Sztajnbok ${ }^{3}$, T. Avcin ${ }^{3}$, R. Cimaz ${ }^{3}$, J. Anton ${ }^{3}$, M. Kostic ${ }^{3}$, T. Lehman ${ }^{3}$, W.-A. Sifuentes-Giraldo ${ }^{3}$ S. Appenzeller ${ }^{3}$, M. Janarthanan ${ }^{3}$, M. Moll ${ }^{3}$, D. Nemcova ${ }^{3}$, M.J. Santos ${ }^{3}$, C. Battagliotti ${ }^{3}$, L. Berntson ${ }^{3}$, J. Brunner ${ }^{3}$, P. Costa Reis ${ }^{3}$, D. Eleftheriou ${ }^{3}$, L. Hare ${ }^{3}$, T. Kallinich ${ }^{3}$, K. Minden ${ }^{2}$, S. Nielsen ${ }^{3}$, Y. Uziel ${ }^{3}$, A. Stevens ${ }^{3}$, C. Pilkington ${ }^{3}$, N. Helmus ${ }^{1} .{ }^{1}$ Hamburg Center for Pediatric and Adolescent Rheumatology, Am Schoen Klinik Eilbek, Hamburg; ${ }^{2}$ German Rheumatism Research Center, Berlin; ${ }^{3} j$ SSc Collaborative Group, Hamburg, Germany

Background: Juvenile systemic scleroderma (jSSc) is an orphan disease, with an estimated prevalence of 3 per 1000000 children. Most jSSc patients primarily present with Raynaud phenomenon (RP). We investigated in our patient of the juvenile scleroderma inception cohort, how fare patients with $(\mathrm{RP}+)$ and without (RP-) RP differed in their clinical presentation at enrolment.

Methods: The jSSc is a prospective cohort of jSSc patients. Patients were enrolled who were diagnosed with jSSc, had a jSSc onset age under 16 years and were younger as age of 18 years at the time of inclusion. The patients are prospectively assessed every 6 months according to a standardised protocol. We reviewed the organ involvement pattern of our patients currently followed in the cohort.

Results: 100 patients are currently followed in the cohort and $89(89 \%)$ of them had RP. The female/male ratio was lower in the RP +group, 3.7:1 compared to $4.5: 1(p=0.808)$. Diffuse subtype was more common in the RP + group, $72 \%$ compared to $63 \%$. Mean age of onset of first non- Raynaud symptomatic was 10.4 years in both groups. Mean disease duration was slightly higher in the RP + group, 3.4 compared to 2.2 years. ANA positivity was higher in the RP + group, $88 \%$ compared to $70 \%(p=0.48)$. Anti-Scl70 was $34 \%$ in the RP +and $20 \%$ in the RP-group $(p=0.34)$. Interestingly $7 \%$ of RP +but none of the RP + were anti-centromere positive. The mean modified skin score was lower in RP +group (mean of $14.8 \mathrm{com}-$ pared to 17.0). There were significantly more nailfold capillary changes $(70 \%$ compared to $18 \%, \mathrm{p}=0.001$ ) and a higher rate of history of ulceration in the $\mathrm{RP}+$ group (49\% compared to $20 \%, \mathrm{p}=0.083$ ). Decreased $\mathrm{DLCO}$ and $\mathrm{FVC}<80 \%$ was higher in the RP-negative group with $45 \% / 50 \%$ compared to $37.5 \% / 31 \%$ respectively. Pulmonary hypertension occurred in $7 \%$ in the $\mathrm{RP}+$ group and there was no case in the RP-group ( $p=0.335)$. RP- group had a higher rate of urinary sediment changes $18 \%$ compared to $4.5 \%$ in the RP +group $(p=0.07)$. No renal crisis or hypertension was reported in neither groups. Gastrointestinal involvement was similar between the two groups with around 35\%. Occurrence of swollen joints was similar in both groups as the frequency of muscle weakness with around $20 \%$. The tendon friction rub occurred around $10 \%$ in both groups. In the patient related outcomes, there was only a difference in rating of Raynauds activity.

Conclusions: The RP-group differed from RP + group in the clinical presentation at enrolment. The absence of Raynaud phenomenon was associated with a decreased rate of history of ulceration, no occurrence of pulmonary hypertension. Interestingly higher rate of urinary sedimentary changes and no anticentromere positivity was observed in RP- patients.

Disclosure of Interest: None declared

DOI: 10.1136/annrheumdis-2018-eular.2913

\begin{tabular}{|l|l}
\hline THU0578 & PATIENTS AND PHYSICIAN RELATED OUTCOMES \\
& IMPROVE SIGNIFICANTLY OVER 12 MONTHS FOLLOW \\
& UP IN PATIENTS WITH JUVENILE SYSTEMIC \\
& SCLEROSIS. RESULTS FROM THE JUVENILE \\
& SCLERODERMA INCEPTION COHORT. WWW.JUVENILE- \\
SCLERODERMA.COM
\end{tabular}

I. Foeldvari ${ }^{1}$, J. Klotsche ${ }^{2}$, M.T. Terreri ${ }^{3}$, M. Katsikas ${ }^{3}$, V. Stanevicha ${ }^{3}$, T. Avcin ${ }^{3}$, R. Cimaz ${ }^{3}$, M. Kostic ${ }^{3}$,W.-A. Sifuentes-Giraldo ${ }^{3}$,F. Sztajnbok ${ }^{3}$, J. Anton $^{3}$, D. Nemcova ${ }^{3}$, M.J. Santos ${ }^{3}$, C. Battagliotti ${ }^{3}$, L. Berntson ${ }^{3}$, J. Brunner ${ }^{3}$, D. Eleftheriou ${ }^{3}$, L. Harel $\left.\right|^{3}$, M. Janarthanan ${ }^{3}$, T. Kallinich ${ }^{3}$, M. Moll ${ }^{3}$, S. Nielsen ${ }^{3}$, V. Smith ${ }^{3}$, K. Torok ${ }^{3}$, A. Stevens ${ }^{3}$, C. Pilkington ${ }^{3}$, N. Helmus ${ }^{1} .{ }^{1}$ Hamburg Center for Pediatric and Adolescent Rheumatology, Am Schoen Klinik Eilbek, Hamburg; ${ }^{2}$ German Rheumatism Research Center, Berlin; ${ }^{3}$ jSSc Collaborative Group, Hamburg, Germany

Background: Juvenile systemic scleroderma (jSSc) is an orphan disease with an estimated prevalence of around 3 per 1000000 children. There are no studies which evaluated prospectively the patient related outcomes in these patients. We report the data from juvenile scleroderma inception cohort (jSSc) regarding organ involvement and patient related outcomes.
Methods: The jSSc is a prospective cohort of jSSc patients. Patients were enrolled who were diagnosed with jSSc, had a jSSc onset age under 16 years and were younger as age of 18 years at the time of inclusion. The patients are prospectively assessed every 6 months according to a standardised protocol. Patients with available 12 months follow up data were included in the analyses. Results: Currently 100 patients are followed in the jSSc cohort. 51 of them had available 12 months follow up data. Among those patients $37(72.5 \%)$ had diffuse and $14(27.5 \%)$ limited subtype. Mean age of onset of disease was $9.5( \pm 4.1)$ years and the mean disease duration at time of inclusion was 3.1 years $( \pm 3.2)$. The proportion of patients treated with DMARD increased from $74.5 \%$ to $88 \%$ at 12 months follow up. $86 \%$ were ANA positive at both assessments. Anti-scl70 positivity increased from $38 \%$ to $42 \%$. Anticentromere antibody positivity was $2.4 \%$ at both assessments. Mean modified skin score decreased from 17.7 to $14.3(p=0.151)$ Raynaud phenomenon occurred in $86 \%$ at enrolment and increased up to $88 \%$ at 12 months follow up. Nailfold capillary changes occurred around $70 \%$ at both assessments, but number of patients with active ulceration decreased from $28 \%$ to $16 \%$ ( $p=0.148)$. The number of patients with decreased FVC (FVC under $80 \%$ ) decreased from $40.5 \%$ to $32 \%$ ( $p=0.497$ ). The number of patients with pulmonary hypertension remained around $10 \%$. No renal crisis or hypertension were reported. The gastrointestinal involvement was around $40 \%$ at both assessments. The number of patients with swollen joints decreased from $24 \%$ to $10 \%(p=0.06)$. The number of patients with muscle weakness decreased significantly from $33 \%$ to $9 \%(p=0.016)$, parallel to the number of patients with elevated $C K$ values which decreased from $27 \%$ to $12 \%(p=0.074)$. All patient related outcomes, like global disease activity $(p=0.048)$, global disease damage $(p=0.05)$, Raynaud activity $(p=0.003)$ and ulceration activity $(p=0.001)$ improved significantly over 12 months. Physician assessed global disease activity $(p=0.003)$ and ulceration activity $(\mathrm{p}=0.001)$ also improved significantly.

Conclusions: Our data show, that jSSc patients over a 12 months disease course stayed quite stable or improved regarding organ involvement. But patient and physician related outcomes regarding activity assessment improved significantly.

Disclosure of Interest: None declared

DOI: 10.1136/annrheumdis-2018-eular.4515

\section{THU0579 VALIDATION OF CONTRAST-ENHANCED MRI SCORES ON (TENO)SYNOVITIS OF THE WRIST IN JUVENILE IDIOPATHIC ARTHRITISPATIENTS BY COMPARISON WITH CHILDREN UNAFFECTED BY CLINICAL ARTHRITIS}

J.M. Van Der Krogt ${ }^{1}$, E.C. van Gulik ${ }^{1}$, R. Hemke ${ }^{1}$, J.M. van den Berg ${ }^{2}$ D. Schonenberg ${ }^{2}$, A. Kindermann ${ }^{3}$, K.M. Dolman ${ }^{4}$, M.A. Benninga ${ }^{3}$, T.W. Kuijpers ${ }^{2}$, M. Maas ${ }^{1}$, C.M. Nusman ${ }^{2}{ }^{1}$ Radiology and Nuclear Medicine, Academic Medical Center, ${ }^{2}$ Pediatric Hematology, Immunology, Rheumatology and Infectious Disease; ${ }^{3}$ Pediatric Gastroenterology and Nutrition, Emma Children's Hospital AMC; ${ }^{4}$ Pediatrics, OLVG Hospital, location West, Amsterdam, Netherlands

Background: Delayed and/or inappropriate treatment of juvenile idiopathic arthritis (JIA) may lead to permanent loss of joint functionality. ${ }^{1}$ Contrast-enhanced MRI is increasingly being accepted as a sensitive tool for detecting JIA disease activity in an early stage. ${ }^{2}$

Objectives: The aim of this study was to assess the validity of two reliable contrast-enhanced MRI scores for the assessment of synovitis and tenosynovitis in the wrist of clinically active JIA patients by a comparison with children unaffected by clinical arthritis.

Methods: An axial T1-weighted MRI sequence with contrast-enhancement and fat-saturation was performed on the wrist of 25 children who had no signs of joint inflammation at clinical examination and who were already subjected to contrast enhanced MR enterography. Wrist MRI scans of 25 clinically active JIA patients were matched based on time-interval between contrast injection and start of the MRI sequence. After being blinded for clinical status, two radiologists scored synovitis and tenosynovitis in consensus. Synovitis was scored at 5 locations by degrees of synovial enhancement (0-2 scale) and synovial inflammation (0-3 scale). Tenosynovitis was scored at the extensor tendons (compartments II, IV and $\mathrm{VI}$ ) and flexor tendons by degree of inflammation based on a $0-3$ scale. ${ }^{3,4}$

Results: Children unaffected by clinical arthritis had significantly lower total synovial enhancement (median $=1$ vs $4, p<0.001$ ) and total synovial inflammation (median $=1$ vs $4, p<0.001$ ) scores compared to clinically active JIA patients (graph). No significant difference in total tenosynovitis score was found between both groups (median $=0$ vs $0, p=0.220$ ). Fifteen out of $25(60 \%)$ clinically active JIA patients were given a total tenosynovitis score of 0 . 